\title{
Exploring the Audit Supervision of China's Medical Insurance Fund Based on the 2019 Medical Insurance Fund Audit Data
}

\author{
Xinyi Zhou ${ }^{1} \&$ Wunhong $\mathrm{Su}^{1}$ \\ ${ }^{1}$ School of Accounting, Hangzhou Dianzi University, Hangzhou, Zhejiang, China \\ Correspondence: Wunhong Su, School of Accounting, Hangzhou Dianzi University, 1158, No.2 Street, Xiasha Higher \\ Education Zone, Hangzhou, Zhejiang 310018, China.
}

Received: May 17, 2021

Accepted: May 28, 2021

Online Published: May 31, 2021

doi:10.5430/afr.v10n2p78

URL: https://doi.org/10.5430/afr.v10n2p78

\begin{abstract}
This study is mainly based on the auditing of medical insurance funds in the audit work report of the National Audit Office's 2019 central and provincial and municipal budget implementation and other fiscal revenues and expenditures to conduct audit supervision of China's medical insurance funds, based on the risk-oriented violation of regulations. Identify and evaluate the risks of each stage of the medical insurance fund from the perspective of risk of breaches, thus draw audit points, summarize the path of the medical insurance fund audit supervision so that that audit resources can be concentrated in important stages and key areas. Audit costs can be reduced, and build the regulatory system of the medical insurance fund audit will be constructed accordingly.
\end{abstract}

Keywords: medical insurance fund, risk-oriented violation of regulations, audit supervision

\section{Introduction}

In recent years, China's demand for social security has been increasing with social and economic development. Medical insurance is an important part of social security, and it is one of the key areas of national concern. At present, China has established the world's largest basic medical insurance network. The number of people participating in basic medical insurance nationwide has exceeded 1.35 billion, and the coverage has stabilized at more than 95\%, basically achieving the full range of insured persons. With the rapid advancement of China's medical insurance system, the Chinese government has made breakthroughs in operation and implementation of medical insurance and the management and use of medical insurance funds. Therefore, to ensure the safe and standard operation of medical insurance funds, China must strengthen the audit supervision of medical insurance funds.

In this study, we analyze the contents of the medical insurance fund audit in the "2019 Budget Execution and Other Financial Revenue and Expenditure Audit Work Report" issued by the Central Government and the Audit Office of 15 provinces and cities to clarify the key subjects and key stages of the medical insurance fund supervision and thus construct a path for medical insurance fund violation risk assessment and audit supervision. Meanwhile, based on clarifying the audit's focus, an audit supervision system for the medical insurance fund was constructed.

\section{Literature Review}

Moral hazard poses a great threat to the safety of the operation, management, and use of medical insurance funds. For example, Zhao et al. (2001) analyze moral hazards in the medical insurance market. It is found that the serious market entities of medical information have broken the transaction balance, and it is very likely to generate moral hazard for various market entities. Thereby market entities such as medical insurance participants and medical institutions may have their own behavioral choices in moral hazard and adversely affect other relevant entities.

$\mathrm{Wu}$ (2014) points out that because the medical moral hazard of all parties involved in medical insurance is inevitable, the only way to reduce the adverse impact of risk can be through strengthening the state's supervision of medical insurance funds and using risk aversion methods. Hu and Zhang (2020) Emphasize that government should play the main role of macro-control in the supervision of medical insurance funds. Xiong (2005) pays more attention to the role of internal control of medical insurance agencies. Medical insurance agencies' establishment of the internal control system is conducive to standardizing business and realizing self-correction, promoting medical insurance fund management safety.

In addition, some scholars emphasize that the state should construct a strict medical insurance audit supervision 
system and pay attention to auditing the compliance, legitimacy, and effectiveness of medical insurance funds. Gaskin(1997) illustrates that the United States constructed a social security fund audit system from 1987 to 1992 to regulate unpaid care and hospital procedures, effectively solving the moral hazard created by trust funds and increasing hospitals' unpaid care by an average of $14.8 \%$. Cost savings for unpaid care in New Jersey increased by $\$ 360$ million.

In summary, although domestic and foreign scholars have a certain degree of explorations in the audit supervision and theoretical research of medical insurance funds, there is relatively little research on medical insurance funds' systematic audit process, and more emphasis is placed on the audit of medical insurance funds. The research on the compliance of fund use and the rationality of related policies rarely lacks in the research literature on the content of the medical insurance fund audit supervision path and audit implementation framework.

\section{Audit Risk Assessment}

\subsection{Clarify Medical Insurance Fund Violation Stages and Outstanding Issues}

In this study, we categorize the outstanding issues related to the current situation of the medical insurance fund audit disclosed in the 2019 audit work report of the Central Government and 15 provinces and cities issued by the National Audit Office into the medical insurance collection stage, the medical insurance management stage or the medical insurance compensation stage.

However, it was discovered that in the medical insurance collection stage, the problems of non-insured coverage, repeated insurance, non-payment, and underpayment were not strictly regulated ( $\mathrm{Bu} \& \mathrm{He}, 2018$ ). And in the medical insurance management stage, the situations of embezzlement of medical insurance funds by management agency or government department, illegal charges by medical institutions, and fraudulent use of information by insured persons, were all not sufficiently disclosed (Joudaki, 2014). Otherwise, there are problems in settlement of arrears of the fund in the compensation payment stage and the unclear review of the expansion of the scope of reimbursement in violation of regulations (Zhu, 2010). Therefore, the above three stages can be subdivided into the medical insurance participation stage, medical insurance payment stage, medical insurance fraud stage, medical insurance arbitrage stage, medical insurance embezzlement stage, and medical insurance reimbursement stage.

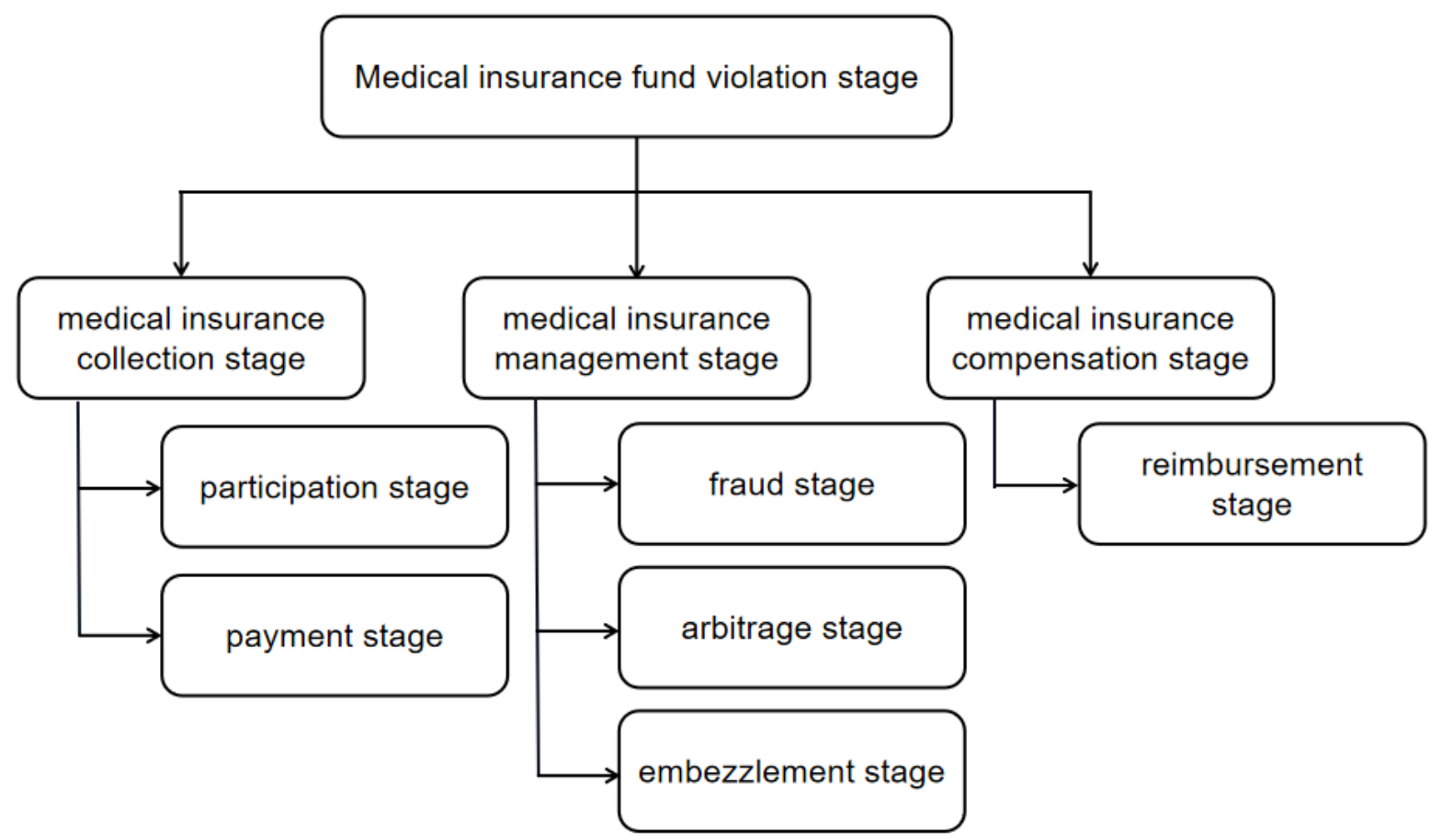

Figure 1. Subdivided medical insurance fund violation stage 


\subsection{Assessment Based on National Medical Insurance Fund Audit Results}

A summary analysis of the main issues disclosed in the audit of the medical insurance fund in the "State Council's Audit Work Report on the Implementation of the Central Budget and Other Financial Revenues and Expenditures in 2019" revealed the disclosed issues are divided according to the violation stages involved. And according to the amount of violation involved in each key violation stage, it determines the key steps of audit supervision. And then, focusing audit resources on key audit steps can greatly help reduce supervision costs and improve audit effects (Garber \& Newhouse, 1999).

In the 2019 National Audit Work Report, China National Audit Office mainly disclosed four types of medical insurance fund audit issues: the medical insurance participation stage, the medical insurance payment stage, and the medical insurance arbitrage stage. Firstly, during the medical insurance participation stage, the evaluation and management of insurance participation are not complete. The situation of cross-regional mobility of personnel enrolling in insurance has not been fully considered. Secondly, part of cities and counties fabricate personnel identities and payment records during the medical insurance payment stage or double-count the number of people. Therefore 8.23 million person-times were overreported by five spot-checked provinces and received an additional 2.364 billion yuan subsidy from the central government. Thirdly, 62 medical institutions and pharmacies and 530 individuals arbitraged 67.12 million yuan by false medical treatments and decomposing hospitalizations during the medical insurance arbitrage stage. Fourthly, during the phase of medical insurance arbitrage, some medical institutions, through repeated charges or over-standard charges, caused the overpayment of 101 million yuan.

Therefore, audit supervision should focus on the problems that may occur during the medical insurance participation process or the medical insurance payment process and the situations such as illegally arbitrage of medical insurance funds by medical institutions.

\subsection{Assessment Based on 15 Provinces' Medical Insurance Fund Audit Results}

In this study, we summarize and analyze the main issues disclosed in the audit of the medical insurance fund in the 2019 audit work report released by the audit offices of 15 Chinese provinces and municipalities according to the stage of violation. On this basis, we summarize the horizontal and vertical data of the violation area and the violation stage of the medical insurance fund. The violation risk of each violation area in each violation stage can be visually compared, and the audit focus should be further analyzed. 
Table 1. Analysis of the stage of violation of medical insurance funds in 15 provinces and cities

\begin{tabular}{ccccccc}
\hline Violation area & $\begin{array}{c}\text { Participation } \\
\text { (number) }\end{array}$ & $\begin{array}{c}\text { Payment } \\
\text { (yuan) }\end{array}$ & $\begin{array}{c}\text { Fraud } \\
\text { (yuan) }\end{array}$ & $\begin{array}{c}\text { Arbitrage } \\
\text { (yuan) }\end{array}$ & $\begin{array}{c}\text { embezzlement } \\
\text { (yuan) }\end{array}$ & $\begin{array}{c}\text { Reimbursement } \\
\text { (yuan) }\end{array}$ \\
\hline Beijing & 485 & 27800 & - & - & - & - \\
Gansu & 6754 & 137244100 & - & 118022700 & - & - \\
Guangxi & 664 & 2868300 & - & 80800 & 111000000 & - \\
Guizhou & 186200 & 15464200 & - & 9893800 & 13760500 & - \\
Hainan & 6606 & - & - & 2831300 & - & 281000000 \\
Henan & 1511 & - & - & 12505200 & - & - \\
Heilongjiang & - & - & 13133700 & 41884100 & - & - \\
Hunan & 629 & 358652600 & 347100 & 1500200 & 91400000 & - \\
Jilin & 1240 & 12941400 & - & - & - & - \\
Liaoning & 9796 & 116498200 & - & 1307400 & - & - \\
Inner & 75895 & - & - & 5870700 & 9838100 & - \\
Mongolia & 1602 & 573620600 & - & - & - & - \\
Qinghai & - & 455400 & 603300 & 3501200 & - & - \\
Shandong & 11000 & - & - & - & - & - \\
Sichuan & 11927 & - & - & 5690000 & - & - \\
Zhejiang & 314309 & 1217772600 & 14084100 & 203087400 & 225998600 & 285151800 \\
total & 1500 & & & - \\
\hline
\end{tabular}

From Table 1, it can be seen that the 15 provinces and cities have the most violations in the medical insurance participation stage, followed by the medical insurance arbitrage stage, and the towns that have violated the medical insurance reimbursement stage are the least. And the highest amount of violations occurred during the medical insurance payment stage. Thus, it can be concluded that there is a greater risk of breaches in the medical insurance participation stage, the medical insurance arbitrage stage, and the medical insurance payment stage, which should be especially focused on at the time of the medical insurance fund audit.

Guizhou Province and Inner Mongolia Province involved the most violations; Gansu Province had the largest breaches during the medical insurance arbitrage stage, followed by Heilongjiang Province. And Qinghai Province had the largest amount of violations during the medical insurance payment stage Province, followed by Hunan Province. Therefore, the provinces and cities with the largest number of violations of the largest amounts involved in the above three steps should be the top priority of audit supervision.

Furthermore, this study analyzes the number of illegal funds involved in the medical insurance audit content in a unified manner. It divides the number of persons in the medical insurance participation stage by the number of violations indicated in the medical insurance participation stage of Inner Mongolia divided by the number of offenders and multiplies the per capital amount of violations by other provinces. The number of violations by the city will receive the number of funds for breaches of the areas. Because only Inner Mongolia indicates both the number of offenders and the number of violations during the medical insurance participation stage, other provinces and cities only show the number of offenders, so to facilitate the calculation, the data from the medical insurance participation stage of Inner Mongolia is used as the calculation standard. In addition, this paper also analyzes the average value of illegal funds and the expected income from illicit activities in different unlawful regions (Attema et al., 2013). And according to the statistics of relevant data, the probability and amount of violation of each stage are weighted and averaged to obtain the expected utility value of a breach of each step. 
Table 2. Analysis of Violation in the Violation Stage of Medical Insurance Fund

\begin{tabular}{cccccc}
\hline Violation stage & Quantity & Probability & Amount & Average & $\begin{array}{c}\text { Violation } \\
\text { expected return }\end{array}$ \\
\hline Participation & 13 & $86.67 \%$ & 142712300 & 10977900 & 123684000 \\
Payment & 9 & $60 \%$ & 1217772600 & 135308100 & 730663600 \\
Fraud & 3 & $20 \%$ & 14084100 & 4694700 & 2816800 \\
Arbitrage & 11 & $73.33 \%$ & 203087400 & 18462500 & 148930800 \\
embezzlement & 4 & $26.67 \%$ & 225998600 & 56499700 & 60266300 \\
Reimbursement & 2 & $13.33 \%$ & 285151800 & 142575900 & 38020200 \\
\hline
\end{tabular}

From Table 2, it can be seen that the number of illegal funds involved in the medical insurance payment stage and the expected income of violations is ranked first when the anticipated revenue of violations is ranked second in the medical insurance arbitrage stage, which also ranks second in the probability of a breach. Although the third-ranked medical insurance participation stage has fewer funds for violations, the likelihood of infringements is greater and to cannot be ignored. Since the order of the expected income of violations takes precedence over the order of the amount of the violation funds, the probability of a breach and the average value of the violation funds, the key stages of audit supervision is the medical insurance payment stage, the medical insurance arbitrage stage, and the medical insurance participation stage.

Combining the analysis results in Table 1 and Table 2, it can be found that when determining the audit focus, if it is a stage of violations with a greater probability, the expected benefits of violations that usually occur at this stage are also relatively large, while those that meet the requirements of the medical insurance participation stage and medical insurance cover. However, since the corresponding violating entity involves all types of violating entities, the key regulatory entities cannot be identified in the stage and the medical insurance payment stage. Therefore, only the provinces and cities involving the largest number of violations or the largest number of people can be identified as key areas for audit supervision if the probability of violation stage is small, which may still correspond to a large number of illegal funds, which usually occurs in the medical insurance embezzlement stage and the medical insurance reimbursement stage. The corresponding violation subjects are mainly management agencies and government departments so that the key issues of audit supervision can be determined. Therefore, according to the expected value, the basic path of audit supervision can be constructed according to the above two situations.

\section{Construct a Medical Insurance Fund Audit Supervision System}

This study starts by identifying and evaluating the risk of violations of medical insurance funds at the above stages, and then construct the medical insurance fund audit supervision system from three levels to guide the audit work to operate more efficiently, which contains institutional level, technical level, and subject level, as the Figure 2 shows. 


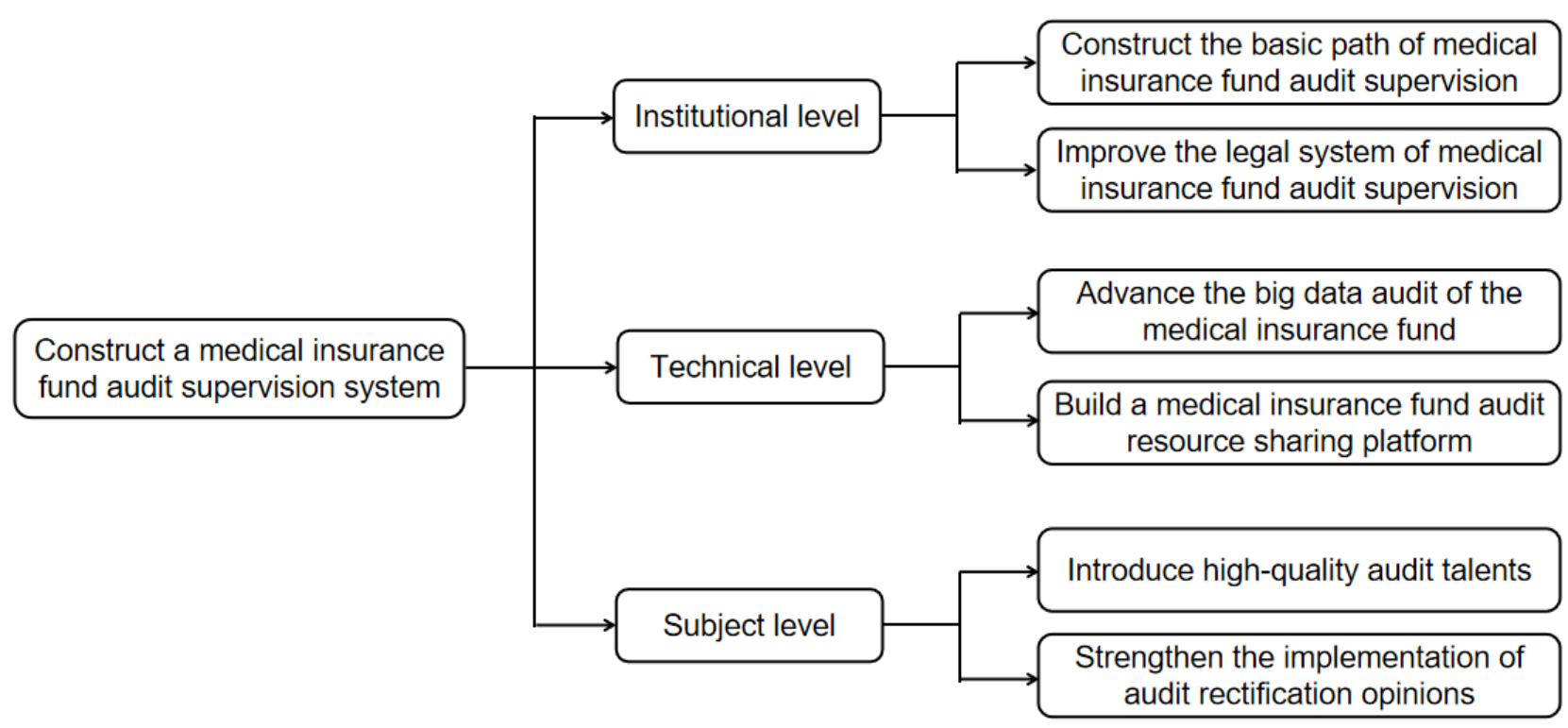

Figure 2. The structure of the medical insurance fund audit supervision system

\subsection{Construct the Basic Path of Medical Insurance Fund Audit Supervision}

Due to the limited human and material resources in the audit, it is impossible to conduct a comprehensive and detailed audit of the whole process of the medical insurance fund. This requires that the audit should be carried out in advance to grasp the key stages and implement supervision so that the violations of the medical insurance fund will be strictly controlled and the risk of infringements Get effective control to ensure that the medical insurance fund is not used in a large amount of illegal use or embezzlement.

From the above analysis, we can see that the basic path of medical insurance fund audit supervision is medical insurance payment stage, medical insurance arbitrage stage, medical insurance participation stage, and medical insurance embezzlement stage, and the earlier the implementation stage, the more audit power should be concentrated on supervision.

First of all, the medical insurance payment stage mainly includes the following. First, the auditors should pay attention to whether the medical insurance fund management agency collects medical insurance funds according to statutory items and basis and whether to expand or reduce the scope of medical insurance funds without authorization. Second, check whether there are no fraudulent acts of concealed or private transfer of fund income. The third is about whether there is a privately set up "small treasury" and whether the late fee is charged according to the regulations, and the bank inquired about the uncollected late fee and made a detailed record.

Next, the medical insurance arbitrage stage mainly includes the following. First, auditors review whether designated medical institutions, private hospitals, and pharmacies have legal and compliance qualifications. Second, they check whether individual pharmacies have monopolistic operations and provide non-prescription medicines. The third is to pay attention to the use of designated medical institutions and pharmacies to decompose hospitalizations, falsely prescribe drugs and other methods to obtain medical insurance funds, review the purchase, sales, and inventory records of drugs and medicinal materials, and pay attention to whether there are large loans with long-term and large amounts of debt, and judge whether there is a problem of falsely prescribing medicines and consumables or replacing items with treatments.

Then, the medical insurance participation stage mainly includes the following. First, review whether the medical insurance-related systems formulated by various localities are consistent with the state's leading policies and pay attention to whether their medical insurance fund coverage includes social assistance objects that are not included. Second, investigate the implementation of various medical insurance systems and the effectiveness of related measures by the local finance departments and local and municipal finance bureaus, and pay attention to whether there are insufficient links between systems in different regions, leading to cross-regional or cross-insurance problems. The third is to target the people in need. Special group registration and participation and implementation of benefits, the list of low-income households, extremely poor supporters, and targeted poverty alleviation personnel 
obtained from the Human Resources and Social Security Bureau, and enterprise retired, difficult enterprise employees, bankrupt enterprise retirees, and laid-off unemployment accepted from enterprises or related institutions For the list of employees and other special groups check whether they have all gone through the insurance payment procedures to participate in the basic medical insurance.

Finally, the stage of medical insurance misappropriation mainly includes the following. First, the audit focus of the auditors is whether the medical insurance fund management agency uses the medical insurance fund by the law and whether there are problems of delay and change in the scope of expenditure during the use of the medical insurance fund. Second, it is to check the use of the medical insurance fund and whether the payment plan is submitted for approval according to legal procedures. The third is to pay attention to illegal transfer, misappropriation, and misappropriation of the medical insurance fund.

\subsection{Improve the Legal System of Medical Insurance Fund Audit Supervision}

Rule-based laws and regulations are the prerequisite and guarantee for the effective conduct of the audit work of China's medical insurance funds. On the one hand, we must formulate comprehensive, specific, and penalized laws and regulations to supervise medical insurance funds. At present, China's legislative work on the audit of medical insurance funds is relatively lagging. Most of the relevant legal documents issued are based on "methods" and "regulations", with relatively low legal effect and deterrence, allowing criminals to take advantage of the legal loopholes. This opportunity also led to the lack of specific and normative guidance for audit work.

On the other hand, it is necessary to formulate the outdated parts of the existing regulations that should be revised. With the development of the medical insurance fund audit work, many earlier legal provisions no longer meet modern development needs. Therefore, it is necessary to promptly abolish or amend the parts that are not compatible with the current work, update and improve the new legal content and new work. The situation adapts. For example, relevant regulations and requirements for computer auditing can be added. At the same time, when revising laws and regulations, the opinions of the people and audit supervision departments should be widely collected to enhance the pertinence and feasibility of rules and policies.

\subsection{Advance the Big Data Audit of Medical Insurance Fund}

The characteristics of high speed, large quantity, and high accuracy presented by big data audit will greatly speed up massive data collection, processing, and analysis, thereby helping to shorten the time for auditors to obtain evidence on-site. At the same time, through the use of rapid and accurate big data analysis, the efficiency of audit supervision is further improved, and the allocation of audit resources is optimized (Feng et al., 2012). Therefore, government audit institutions should make full use of the characteristics of big data auditing speed, large number, and high accuracy, and organize big data audit experts to form a data analysis team to carry out big data analysis. Quickly and accurately discover audit doubts and focus on audits, provide the audit team with major doubts or case clues, overcome the shortcomings of traditional audit methods, improve audit supervision efficiency, expand audit supervision coverage, and prevent audit risks more effectively and timely and shortening the audit cycle.

\subsection{Build a Medical Insurance Fund Audit Resource Sharing Platform}

Build a medical insurance fund audit resource sharing platform to realize information resource sharing (Shi, 2015) and help government audit institutions adopt more targeted audit procedures to correct errors, check frauds, control audit risks, and improve service quality and efficiency audit work. The management system and personnel arrangement of the medical insurance fund management agency, the internal control and implementation of the government audit agency, the income, and expenditure and investment operation of the medical insurance fund audit, the detailed information of the audit situation over the years, the implementation of the rectification of audit issues, etc., are unified into the platform Management not only facilitates the sharing of audit experience by auditors, avoids repeated audits and waste of resources, but also improves the quality and efficiency of performing audits and obtaining audit evidence, thereby promoting the in-depth development of full audit coverage. At the same time, it is also convenient for normalized inspection of medical insurance fund audit resources to ensure that system loopholes in the management and operation of medical insurance funds can be discovered and corrected promptly. The governance effect of audit supervision is further improved.

\subsection{Introduce High-Quality Audit Talents}

The current audit talent management model in China mainly has shortcomings. For example, the talent structure is too single, talent introduction and training are relatively weak, and the performance evaluation mechanism of auditors is not yet perfect. At the same time, China's medical insurance system is developing rapidly, requiring further improvement of the professional skills and overall quality of medical insurance fund auditors. 
Through the establishment of a composite medical insurance fund audit team and establish a talent introduction method that meets the characteristics of medical insurance fund audits, the introduction of talents through multiple channels and multiple disciplines, and the absorption of skills in various professional fields can promote the complementary advantages of audit personnel and enhance Regulatory power of medical insurance funds. For example, you may encounter professional problems related to law, finance, investment, insurance, and other fields in the medical insurance fund audit process. Introducing or hiring professionals in various related fields to assist in the audit work improves medical care. The efficiency of insurance fund audit work has promoted the comprehensive and smooth progress of the audit work.

\subsection{Strengthen the Implementation of Audit Rectification Opinions}

After the medical insurance fund audit department discovers the problem and proposes a targeted audit rectification opinion, the relevant responsible management department should quickly intervene and correct the problem to avoid further losses. At the same time, the funds that have been misappropriated or used in violation of regulations must be fully recovered. At the same time, relevant management departments also need to publicize and notify the violation cases found in the entire industry, which will help self-examination and self-correction of similar violations and serve as a warning.

In addition, reasonable audit recommendations are crucial to the audit and reform of the medical insurance fund. Therefore, when the auditors make audit recommendations, they should pay attention to improving the audit recommendations they put forward. On the one hand, it is necessary to ensure that the proposed audit recommendations are pertinent. The rectification opinions must include solutions to various problems that occurred during the operation of the medical insurance fund and analyze the internal causes of multiple problems; on the other hand, ensure that the proposed audit recommendations are feasible. If the content of the audit rectification opinions is too broad or is not executable, then the statements can only be purely formal and cannot play any substantive role.

\section{Conclusion}

In the paper, we analyze China's medical insurance fund's current audit status and outstanding issues by studying the theoretical basis of medical insurance fund auditing and domestic and foreign literature, combined with the 2019 audit work report published by the China National Audit Office. By sorting out the entire audit process of the medical insurance fund, we classify the outstanding issues disclosed into the six medical insurance fund subdivision stages we have established, and use data statistics to determine the size of the violation risk and the probability of violation, the amount of a breach, and the violation of regulations at each stage. The expected return is proportional, and the expected return from violation has the greatest impact on the risk of a breach. This study determines the key stages and supervision paths of the medical insurance fund audit work to realize the concentration of limited audit resources to focus on the audit at the key non-compliance stage, reduce audit costs, and improve audit efficiency and resource utilization. Finally, in response to the existing problems in the audit work of China's medical insurance fund, this article proposes to build a medical insurance fund audit supervision system, including strengthening system construction, innovating audit technology, and improving the professional quality of auditors, etc., hoping to help the further development of the audit work of China's medical insurance fund.

Since there are few related data disclosed in the audit work of the medical insurance fund, the relevant data analysis in the study is relatively small, and the salient issues of the audit are not comprehensive enough. In addition, due to the limitations of relevant audit practice experience, the depth and breadth of the research in this article are quite lacking, and the audit supervision system constructed is not mature enough. However, we still hope that the paper's content can provide new ideas and references for the development of audit work.

\section{References}

Joudaki, H., Rashidian, A., Minaei-Bidgoli, B., Mahmoodi, M., Geraili, B., Nasiri, M., \& Arab, M. (2014). Using data mining to detect health care fraud and abuse: a review of the literature. Global journal of health science, 7(1), 194-202. https://doi.org/10.5539/gjhs.v7n1p194

Hu, Z., \& Zhang, W. (2020). The Research on Audit \& Supervision System of Medical Insurance Fund in China. China Health Insurance, 04, 29-32. https://doi.org/10.19546/j.issn.1674-3830.2020.4.010

Davis, E. P., \& Haan, L. D. (2012). Pension fund finance and sponsoring companies. Journal of Pension Economics and Finance, 11(3), 439-463. https://doi.org/10.1017/S1474747212000030

Wang, C. (2020). Design and Application of Network Audit System of Social Security Fund. Communication of Finance and Accounting, 17, 145-148. https://doi.org/10.16144/j.cnki.issn1002-8072.2020.17.032 
Gillion, C. (2010). The Development and Reform of Social Security Pensions: the Approach of the International Labour Office. International Social Security Review, 53(1), 35-63. https://doi.org/10.1111/1468-246X.00062

Du, Y., \& He, Y. (2018). Research on the Countermeasures to Strengthen the Audit and Supervision of Medical Insurance Funds. Audit Monthly, 09, 16-18. https://doi.org/10.15882/j.cnki.sjyk.2018.09.005

Shi, X. (2015). Analysis on the Impact of Big Data Era on Social Security Audit. China Economist, 02, 163-164. https://doi.org/10.3969/j.issn.1004-4914.2015.02.073

Gaskin, D. J. (1997). Altruism or moral hazard: the impact of hospital uncompensated care pools. Journal of Health Economics, 16(4), 397-416. https://doi.org/10.1016/S0167-6296(96)00539-5

Guo, Z. Y. (2017).Health Insurance and the Demand for Medical Care: a Case Study from China. Asian Journal of Economics and Empirical Research, 2017, 4(1), 8-13. https://doi.org/10.20448/journal.501.2017.41.8.13

Garber, A. M., Culyer, A. J., \& Newhouse, J. P. (1999). Advances in cost-effectiveness analysis of health interventions. Handbook of Health Economics, 01, 181-221. https://doi.org/10.1016/S1574-0064(00)80163-8

Gendron, Y., Cooper, D. J., \& Townley, B. (2001). In the name of accountability - state auditing, independence, and new public management. Accounting Auditing \& Accountability Journal, 14(3), 278-310. https://doi.org/10.1108/EUM0000000005518

Zhao, X., Wang, Y., Song, Q., \& Wang, J. (2001). Moral Hazard and Prevention in the Basic Medical Insurance for Urban Employees in China's Country. Health Economics Research, 07, 17-19. https://doi.org/10.14055/j.cnki.33-1056/f.2001.07.008

Feng, W., Wei, M., Long, J., \& Yan, Y. (2012). Study on Computer-Assisted Audit and its Applications. Applied Mechanics and Materials, 380-385. https://doi.org/10.4028/www.scientific.net/AMM.198-199.380

Zhu, Q. (2010). Research on the Problems and Countermeasures of Social Security Audit in the Information Environment_ Based on the Analysis of the Application of Modern Audit Technology in Social Security Audit. Auditing and Finance, 06, 22-23. https://doi.org/10.19419/j.cnki.36-1264/f.2010.06.011

Rao, D., \& Su, Z. (2019). Research on the Supervision of Pension Funds from the Perspective of Audit. Communication of Finance Accounting, 34, 79-83. https://doi.org/10.16144/j.cnki.issn1002-8072.2019.34.018

Xiong, M. (2005). Difficulties and Countermeasures of China's Medical Insurance System Reform. International Medicine and Health Guidance News, 11, 23-27. https://doi.org/10.3760/cma.j.issn.1007-1245.2005.11.006

Li, Z. (2016). Audit Thinking on Integrating Urban and Rural Medical Insurance System Obstacles. Auditing and Finance, 10, 36-37. https://doi.org/10.19419/j.cnki.36-1264/f.2016.10.015

Hu, G., Shi, H., \& Tan, J. (2013). Research on the Reform of Social Security Auditing under the Background of Economic Transition. Finance and Accounting Monthly, 08, 98-101. https://doi.org/10.19641/j.cnki.42-1290/f.2013.08.031

Wu, C. (2014). Research on Policy Incentive Paths to Promote the Sustainable Development of Health Insurance from the Perspective of Prospect Theory. Macroeconomics, 11, 127-137. https://doi.org/10.16304/j.cnki.11-3952/f.2014.11.002

Attema, A. E., Brouwer, W., \& L'Haridon, O. (2013). Prospect theory in the health domain: a quantitative assessment. Journal of Health Economics, 32(6), 1057-1065. https://doi.org/10.1016/j.jhealeco.2013.08.006

\section{Copyrights}

Copyright for this article is retained by the author(s), with first publication rights granted to the journal.

This is an open-access article distributed under the terms and conditions of the Creative Commons Attribution license (http://creativecommons.org/licenses/by/4.0/). 